International Journal of Biomedicine I June 2019 - Volume 9, Issue Suppl_1: Abstracts From the Second Russian International Conference "Cryo-electron microscopy 2019: achievements and prospects"

POSTER ABSTRACT PRESENTATIONS

SESSION TITLE: STRUCTURE OF MEMBRANE PROTEINS

DOI: 10.21103/IJBM.9.Suppl_1.P10

\title{
Abstract P-10: Comparison of Ion Coordination in Similar Selectivity Filters of Bacterial Sodium and Eukaryotic Calcium Voltage-Gated Channels
}

\author{
Ivan N. Terterov ${ }^{1}$, Alexey A. Bogdanov ${ }^{1}$, Ilya A. Pozdnyakov ${ }^{2}$ \\ ${ }^{1}$ Saint-Petersburg Clinical Scientific and Practical Center of Specialized Types of Medical Care \\ (Oncological), St. Petersburg, Russia; ${ }^{2}$ Institute of Cytology RAS, St. Petersburg, Russia
}

Background: Prokaryotic voltage-gated sodium channels (Navs) and eukaryotic voltage-gated calcium channels (Cavs) are the members of the same superfamily. Bacterial Navs are homotetramers with a selectivity filter formed by four segments given form a P-loop of each monomer. In contrast, eukaryotic Cavs are pseudotetramers with four asymmetric but homologous P-loops and probably evolved from prokaryotic Navs by two rounds of duplication. Nevertheless, selectivity filters of bacterial Navs and eukaryotic Cavs are very similar, and surprisingly, they have identical E/E/E/E high-field-strength (HFS) site. Since HFS site was considered to be responsible for ion selectivity, the mechanism of different $\mathrm{Na} / \mathrm{Ca}$ discrimination of these channels is still unclear.

From a comparison of the new cryo-EM structure of mammalian Cav1.1 channel with X-ray structure of bacterial Nav, it is clear that backbone positions of Glu residues of HFS sites are identical in these two channels but the side chains have different geometries. However, it is unclear whether these side chain structures are just a distinct conformers from the same ensemble, or their difference is functional and defines dissimilar channel selectivity.

Methods: In order to explore conformational motions of the HFS site residues we conducted molecular dynamics simulations of NavMs channel (PDB: 5BZB) and Cav1.1 channel (PDB: 6BYO) in the same conditions with single ion coordinated in the selectivity filter.

Results: We found that carboxylate groups of Glu residues of HFS site coordinate partially hydrated cation in the pore lumen, as was demonstrated before. A closer look shows that during ion coordination Glu side chains notably alter their conformations from symmetric geometry of X-ray NavMs structure towards asymmetric one observed for Cav1.1 with cryo-EM.

Conclusion: Each published channel structure captures only one Glu side chain geometry in HFS site, while it is thought that these groups sample multiple conformation when ion path through the pore. Results of our simulations suggest that side chains of E/E/E/E HFS sites of NavMs and Cav1.1 channels are flexible and may adopt similar conformations when coordinate the same cation. 
Key Words: voltage-gated ion channels $\bullet$ Cavs $\bullet$ Navs

Sources of Funding: This work was supported by RFBR grant No 18-34-00992.

International Journal of Biomedicine. 2019;9 Suppl 1: S20-21. doi: 10.21103/IJBM.9.Suppl_1.P10

C2019 International Medical Research and Development Corporation 